\title{
Minor modifications in obtainable Arabidopsis floral dip method enhances transformation efficiency and production of homozygous transgenic lines harboring a single copy of transgene
}

\author{
Priyanka Das, Naveen Chandra Joshi*
}

Institute of Life Sciences Bhubaneswar Orissa India

School of Life Sciences Jawaharlal Nehru University New Delhi-110067 India.

Email: *ncjjbiotech@gmail.com

Received 10 January 2011; revised 16 January 2011; accepted 20 January 2011.

\begin{abstract}
Many researchers have developed various methods for in-planta or floral dip transformation of Arabidopsis thaliana, one of the simple protocol and widely used to produce transgenic Arabidopsis. As the efficiency and ease of getting a transformant is very much time consuming effort and less number of the transformants people get, we have developed a little modified transformation protocol to avoid the disparities. Four types of inoculums (inoculum-1, inoculum-2, inoculum-3 and inoculum-4) were used to check the transformation efficiency out of which Inoculum-3 showed the highest rate of transformation among the four types. $0.07 \%$ Twin-20 also acts in same manner as silwet L-77 to increase the rate of transformation efficiency and glucose instead of sucrose can be used in inoculum to transform Arabidopsis. After vacuum infiltration keeping the Agrobacterium infected plants for 7 - 8 hrs horizontally in low light at $28^{\circ} \mathrm{C}$ temperature condition, considered best to get an increased number of transformed seeds. Modified protocol produced $\sim 12 \%-14 \%$ increase in transformants. Selection pots (kanamycin supplemented soil filled pots) in place of selection plates (Kanamycin supplemented Murashige and Skoog agar plates) proved beneficial as no MS medium and no aseptic condition is required for selection of transformed plants. This increase in transformation efficiency consequently increased the percentage of homozygous and single copied stable transgenic lines.
\end{abstract}

Keywords: Arabidopsis Thaliana; Floral-Dipping; Copy Number; Homozygous; Inoculum; Transgenic Plant

\section{INTRODUCTION}

Efficient study of plant gene function has been permissi- ble through transgenic approaches. Plant transformation is a process of genetic operation by which foreign genes are introduced into plant genome and stably integrated and the transformed cells are regenerated to get transgenic plants. A plant transformation method that excludes the use of tissue culture and plant regeneration would greatly reduce the time required to produce transgenic plants, and such a method was first described as "in planta" transformation almost 20 years ago [1] Much effort has been given to get stable transgenic lines. Feldmann and Marks [1] co-cultivated germinating seeds of Arabidopsis thaliana with an Agrobacterium tumefacien strain harboring a disarmed Ti plasmid and a binary vector, and remarkably, they got stable transgenic lines, although the transformation rates were very low. Several years later, the Pelletier group infiltrated flowering Arabidopsis plants with Agrobacterium and dramatically improved the transformation efficiency [2]. This account of transformation approach was frequently named as the "Agrobacterium vacuum infiltration method" [3]. Briefly, it involves uprooting of flowering Arabidopsis plants, vacuum infiltration of the plants using an Agrobacterium cell suspension, re-planting, harvesting of seed several weeks later, and screening for primary transformants on medium containing the appropriate selective agent (usually an antibiotic or a herbicide). These transformation procedures were later simplified and substantially improved [4]. The vacuum-aided infiltration of inflorescence [2] was substituted using a surfactant (Silwet L-77), which had already been shown, in the formulation of some pesticides, to help chemicals enter the plant tissues. All these modifications simplified the initial procedure. The Arabidopsis flower buds were simply dipped in an Agrobacterium cell suspension containing 5\% sucrose (wt/vol) and $0.01 \%-0.05 \%$ Silwet L-77 (vol/vol) 
to allow uptake of the Agrobacteria into female gametes $[5,6]$. This simple transformation plan was commonly known as "the floral dip method" [4]. It requires minimal labor, relatively inexpensive equipment and few specialized reagents, and can be successfully performed. The floral dip method easily maintains genomic stability in Arabidopsis thaliana transgenic plants, which can otherwise be harmed by the tissue culture-based transformation [7]. The success and popularity of this floral transformation procedure is reflected by its high citation index since its description $[3,4]$. Similar to floral dip, floral spray also works very well with Arabidopsis [8]. Using a similar protocol, Curtis and Nam reported the successful production of transgenic radish and Brassica rapa [9], Arabidopsis lasiocarpa [10] and rape seed Brassica napus [11].

The segregation test of transgene was useful to identify transgenic plants with single locus DNA integration and zygosity determination. Southern blot analysis determines the copy number of the transgene. But it is both time and labor consuming and precautions were required at the step of radioactive handling. The quantitative RT-PCR and single step PCR method could able to overcome those types of problems in identifying copy number analysis.

Our wide use of the floral dip method over the years to generate transgenic Arabidopsis plants in the laboratory has allowed us to adjust its various steps. However, there is scope to improve this protocol. Many researchers have put their hands on modification of the previous protocols to improve transformation process $[8,12-15]$. There are some steps that we found to be both time-consuming and costly. We provide some modifications in steps of floral-dip method of Arabidopsis that eradicates the need to screen on sterile conditions as only agro-peat soil treated with kanamycin is a suitable alternative to an agar substrate during the seed selection process. In another development, we provide a description of modified inoculum preparation for infiltration that supports direct dipping and plant transformation, thereby enhancing the chance of more transformant production having homozygous and single copy of integrated target gene.

\section{MATERIAL AND METHOD}

\subsection{Materials}

Plant

\author{
Arabidopsis thaliana (Columbia-0) (Figure 1(a)) \\ Bacterium \\ . Agrobacterium strain: GV1301 \\ Chemicals and Reagents \\ - agarose.
}

- MS medium

- Liquid LB medium (10 g tryptone, $5 \mathrm{~g}$ yeast extract,

$10 \mathrm{~g} \mathrm{NaCl}$ per liter)

- sucrose

- glucose

. $0.025 \%, 0.05 \%, 0.075 \%$ and $0.1 \%$ (vol $/ \mathrm{vol})$ Silwet

L-77 (Lehle Seeds)

- Selection plates (see reagent setup)

- Kanamycin

. ethanol

- Tween-20

. Ethidium bromide

. Standard PCR buffers and solutions.

\subsection{Equipments}

. Growth chambers or light room or greenhouse, adjustable to long-day condition of $16 \mathrm{~h}$ light $/ 8 \mathrm{~h}$ dark, $20 \pm$ $2^{0} \mathrm{C}$

$.30^{\circ} \mathrm{C}$ chamber

. Pots for plant growth

. Laminar-Air flow hood

- Acrodisc Syringe Filter with 0.2-mm membranes

(Pall Life Sciences)

. Petri dishes: $150 \times 150 \times 25 \mathrm{~mm}$ (Falcon 3025)

. Agarose gel running system

. Thermal cycler

\subsection{Reagent Setup}

\section{MS medium and selection plates}

. Autoclaved MS medium (4.3 g Murashige \& Skoog salts, $10 \mathrm{~g}$ sucrose, $0.5 \mathrm{~g}$ MES, $8 \mathrm{~g}$ agar per liter; $\mathrm{pH}$ 5.7), cooled to approximately $50^{\circ} \mathrm{C}$ before pouring into Petri dishes (MS solid medium). To prepare selection plates $50 \mathrm{mgL}^{-1}$ kanamycin was added to MS medium.

\section{Selection pots}

- Autoclaved soil (agropeat)-To prepare selection pot, $50 \mathrm{mgL}^{-1}$ kanamycin was supplemented to the pot filled with soil until rosette leaves appeared.

\section{Buffers}

. TAE buffer for agarose gel running.

\section{Primers}

- Kanamycin gene (transgene) specific and a known (control) single copy gene specific forward and reverse primers were used (Table 2)

\subsection{Methodology}

\section{Step 1: Growing Arabidopsis plants}

Arabidopsis seeds were kept for 3 days at $4^{0} \mathrm{C}$ to break dormancy. Vernalized seeds were layered on agro-peat filled pots. Pots were placed and well watered and allowed to grow in growth chamber or green house in long day $(16 \mathrm{hL} / 8 \mathrm{hD})$ condition up to inflorescence or floral stage comes up (Figure 1(a)). 
Table 1. Composition of inoculums.

\begin{tabular}{c|c|c|c|c}
\hline Composition & Inoculum-1 & Inonulum-2 & Inoculum-3 & Inoculum-4 \\
MS salt & $0.5 \mathrm{X}$ & $0.5 \mathrm{X}$ & $0.5 \mathrm{X}$ & $0.5 \mathrm{X}$ \\
$\mathrm{B}_{5}$ vitamins & $1 \mathrm{X}$ & $1 \mathrm{X}$ & $1 \mathrm{X}$ & $1 \mathrm{X}$ \\
Glucose & --- & -- & $5 \%$ & $5 \%$ \\
Sucrose & $5 \%$ & --- &.-- \\
Silwet L-77 & $0.03 \%$ & ---- & $0.075 \%$ & $0.1 \%$ \\
Tween-20 & --- & $0.075 \%$ & ---- & -- \\
\hline
\end{tabular}

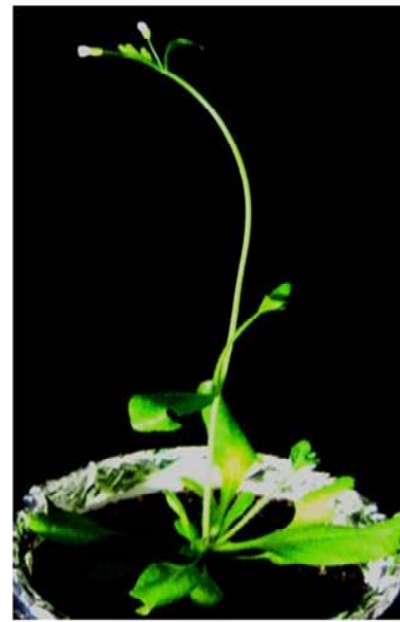

(a)

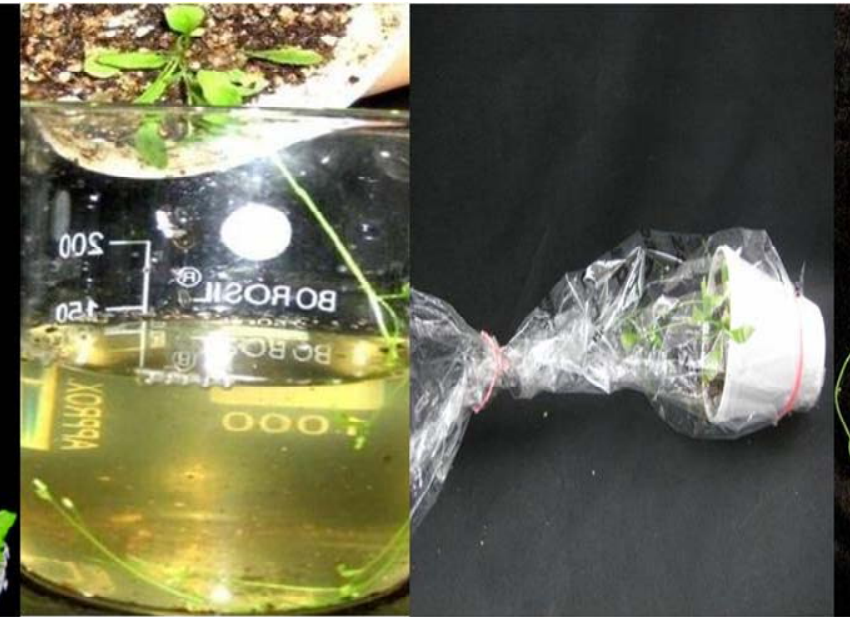

(b) (c)

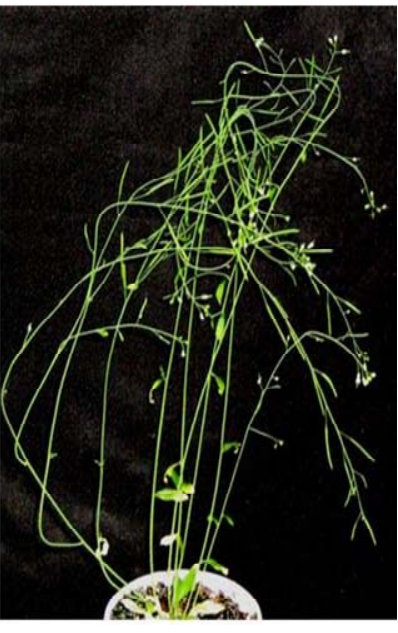

(d)

Figure 1. Steps of floral dip transformation of Arabidopsis thaliana. (a) Plant at budding stage; (b) Floral Dip in Agrobacteria culture by inverting the plant; (c) Incubation of plant horizontally to ground after dipping; (d) Matured $\mathrm{T}_{0}$ plants.

\section{Modifications in Step 1:}

No net or nylon screen was used which proved economically reliable and less labor consuming. The tightness of soil was considered adequate to keep it as such at the time of floral dip. A very little loss of soil was occurred at the time of vacuum infiltration (Figure 1(b)). Clipping of primary inflorescence is not required as this reduces the time to get ready with the floral buds for infiltration.

\section{Step 2: Agrobacteium culture and inoculum prepa-} ration

. Agrobacterium strains that harboring gene of interest in a binary vector (pCAMBIA 1304) were inoculated in a $5 \mathrm{ml}$ culture tube and incubated at $28^{\circ} \mathrm{C}$ and $200 \mathrm{rpm} / \mathrm{min}$ rotation for $48 \mathrm{hrs}$. This is the primary culture.

. Secondary culture was prepared by inoculating $1 \mathrm{ml}$ of primary culture into $1000 \mathrm{ml}$ of LB medium supplemented with specific antibiotic and allowed to grow up to $\sim 2.4-2.5 \mathrm{OD}$ at $600 \mathrm{~nm}$ (stationary phage), in same condition as primary culture.

- Bacteria cells were collected by centrifugation at $5000 \mathrm{xg}$ and the inoculum was prepared by re-suspending the pellet in infiltration medium which is composed of $0.5 \mathrm{X}$ MS salts, $1 \mathrm{X}$ B5 vitamins, $5 \%$ sucrose or glucose, $0.004 \mathrm{M}$ BAP, $0.02 \%-0.1 \%$ (vol $/ \mathrm{vol})$ silwet L-77 or
$0.1 \%$ tween- 20 and $\mathrm{pH}$ adjusted to 5.7 .

\section{Modifications in Step 2:}

.We went up to $\sim 2.5$ OD of the bacterial culture to check its effect on the transformation rate.

.We used glucose rather than sucrose in one of the inoculums (Table 1).

.We used $0.7 \%$ (vol $/ \mathrm{vol})$ tween-20 rather than silwet L-77 in one of the inoculums (Table 1).

. Four types of inoculums were used in this study (Table 1)

\section{Step 3: Floral dipping and vacuum infiltration}

- Plants in the stage of budding without clipping of primary inflorescence were taken and dipped in the inoculums by upturning. One plant (Figure 1(b)) or multi plants per pot can be used to do the experiment. The entire set up was kept in a bell jar and the vacuum (400 $\mathrm{mm} \mathrm{Hg}$ ) was applied till bubbles were formed on the leaves and stem. The vacuum was rapidly released and plants were left in liquid for 5 minutes.

. The plants were removed from the bacterial suspension; the inflorescence part was covered with poly bag (to maintain humidity) and placed parallel to ground (Figure 1(c)) for $7-8 \mathrm{hrs}$ at $28^{\circ} \mathrm{C}$. The plants were uncovered after $7-8 \mathrm{hrs}$ and rinsed with water to wash the excess silwet L-77 or tween-20 and allowed to grow photoperiodically $(16 \mathrm{~h} \mathrm{~L} / 8 \mathrm{~h} \mathrm{D})$ in plant growth chamber 
in $100 \mu$ moles photons $\mathrm{m}^{-2} \mathrm{~s}^{-1}$ of light intensity.

- Watering was stopped at the starting time of pod maturation (Figure 1(d)). Seeds were collected after the pods turned to brown.

- Seeds can be stored at room temperature up to $3-4$ months or in refrigerator up to 1 year.

Step 4: Screening of $\mathbf{T}_{\mathbf{0}}$ transformed seeds

- Seeds were surface sterilized under laminar hood with $70 \%$ ethanol, washed 4 times with sterile water and plated by pipetting method on MS plate (selection plate) containing specific antibiotic (kanamycin in our case) or vernalized seeds were directy layered on antibiotic containing agopeat (soil) .

. $\sim 150$ seeds were plated for antibiotic selection on each plate or pot.

- Plates were dried up under laminar flow, sealed and kept three days in $4^{0} \mathrm{C}$ for vernalization.

- Following vernalization they were moved to growth chamber having long day condition and $22^{\circ} \mathrm{C}$ temperature for germination and growth.

\section{Modifications in Step 4:}

. $\mathrm{T}_{0}$ seeds ( 150 seeds) were vernalized and layered on an agropeat filled soil which was pretreated with antibiotic solution, without any surface sterilization.

. Soil was regularly treated with antibiotic solution until rosette leaves come up.

\section{Step 5: Genomic DNA isolation}

Genomic DNA was isolated by CTAB method [16] .Small piece of leaf tissue $(1 \mathrm{~cm} \mathrm{X} 1 \mathrm{~cm})$ of primary transformants was ground with genomic DNA extraction buffer (2\% CTAB, 1.4M NaCl, 20 mM EDTA, $100 \mathrm{mM}$ Tris- $\mathrm{Cl}$ and $0.1 \% \beta-\mathrm{ME}$ ) at room temperature and kept at $60^{\circ} \mathrm{C}$ in a water bath for $30 \mathrm{~min}$. To this phenol: chloroform: isoamyl alcohol (24:24:1) was added and mixed by vortexing followed by centrifugation at maximum speed for $5 \mathrm{~min}$ in a microfuge at room temperature. The aqueous layer was transferred to another fresh tube. To the aqueous phase 2/3 volume of isopropanol was added, mixed properly and kept at room temperature for $5-10$ min to precipitate the genomic DNA. After precipitation of genomic DNA samples were centrifuged at maximum speed for $5 \mathrm{~min}$ at room temperature in a microfuge. The pellet was washed 3 times with $70 \%$ ethanol, dried and dissolved in $30 \mu \mathrm{l}$ sterile water containing $20 \mu \mathrm{g} / \mathrm{ml}$ RNase and incubated at $37^{\circ} \mathrm{C}$ for $30 \mathrm{~min}$ and used for analysis.

Step 6: Confirmation of T-DNA integration in Arabidopsis genome

To detect the integration of T-DNA, PCR was performed with kanamycin specific forward and reverse primers (Table 2) amplifying 800bp.

Step 7: Segregation analysis and detection of homozygous stable transgenic lines

- Segregation analysis was performed to estimate the number of copies of transgene that integrated into the genome of transgenic Arabidopsis. Genomic DNA was isolated from the leaves of $T_{1}, T_{2}$ and $T_{3}$ generation plants, produced from $\mathrm{T}_{0}$ lines dipped in inooculum 1 and inoculum-3. Gene specific primers were used for PCR analysis and thermal cycling conditions were similar, as described by Fu et al [17].

Step 8: Identification of Single copied transformants

Transgenic lines carrying single copied transgene were identified by single step genomic PCR method described by Kihara et al [11]. About $20 \mu \mathrm{g}$ of genomic DNA was used as templates in a total volume of $20 \mu \mathrm{l}$ of PCR mixture in individual PCR tube. The PCR mixture consisted of Taq DNA polymerase, dNTPs and $0.5 \mu \mathrm{M}$ each of primer pairs targeting the transgene and a known single copy control gene (4-hydroxyphenylpyruvate deoxygenase-[4HPPD;At4g03280]). The PCR reaction was carried out with up to 22 cycles of $94^{\circ} \mathrm{C}$ for $30 \mathrm{~s}, 60^{\circ} \mathrm{C}$ for $30 \mathrm{~s}$ and $72^{\circ} \mathrm{C}$ for $1 \mathrm{~min}$. After PCR, samples were loaded onto regular agarose gel $(1.5 \% \mathrm{w} / \mathrm{v})$ and visualized by ethidium bromide staining. Picture of gel was taken by gel documentary system-(Bio-Rad) and the band intensity was quantified using NIH image software (http://rsb.info.nih.gov/nih-image). Ratio of the band intensity of target and control was calculated to determine the copy number of the transgene.

\section{RESULT}

Inoculum type for vacuum infiltration and antibiotic screening of $\mathrm{T}_{1}$ transformants:

Table 2. Sequences and Tm values used in Quantitative Dual Target PCR for determination of copy number of T-DNA integrated into the Arabidopsis thaliana genome. 4HPPD, represents 4-hydroxyphenylpyruvate dioxygenase.

\begin{tabular}{ccccc}
\hline Gene & Template & Primer name & Primer sequence $\left(\mathbf{5}^{\prime}-\mathbf{3}^{\prime}\right)$ & Tm \\
\hline Control & Genomic DNA & 4HPPDF' & ATGTGTCTATCGTTAGCTTCTACAGCT & 59.20 \\
& & & & \\
Control & Genomic DNA & 4HPPDR' & CTCTGCGAATTGGTGAAAACC & 60.0 \\
Transgene & Genomic DNA & KanamycinF' & TCGACCATGGGGATTGAAC & 60.10 \\
\multirow{2}{*}{ Transgene } & \multirow{2}{*}{ Genomic DNA } & KanamycinR' & TCGACCATGGGGATTGAAC & 60.10 \\
\hline
\end{tabular}




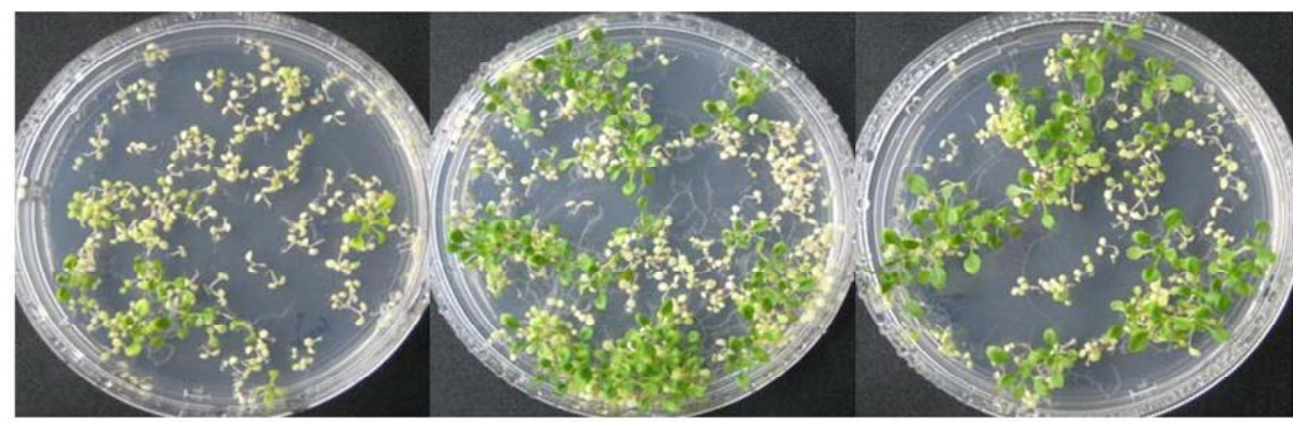

(a)

(b)

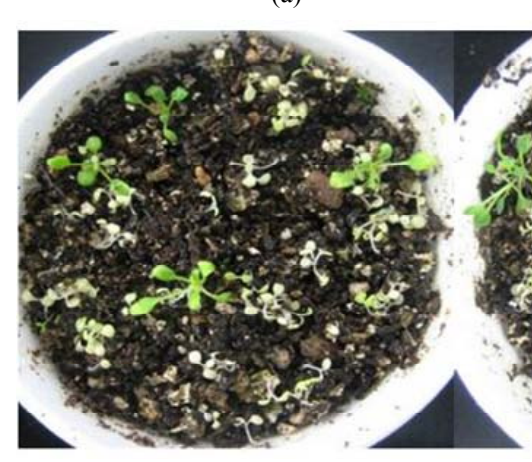

(d) (c)

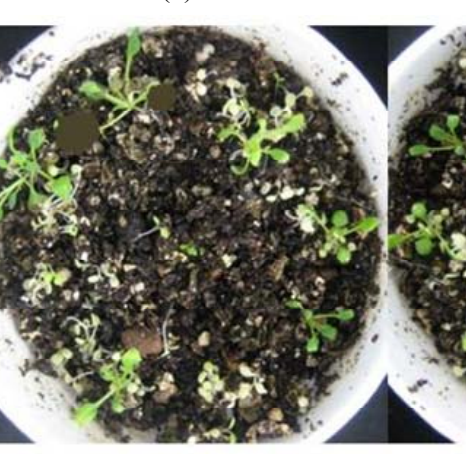

(e)

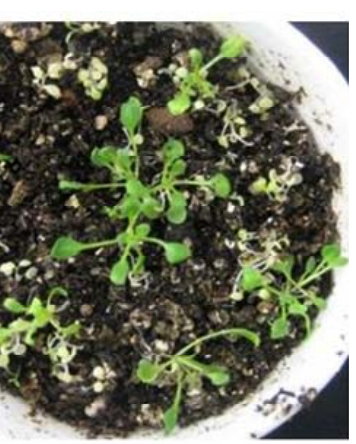

(f)

Figure 2. Screening of kanamycin resistant $T_{1}$ plants. (a), (b) and (c) showing screening of $T_{1}$ transformants of inoculum-1, 2 and 3 dipped plants respectively, on kanamycin containing MS agar plates. (d), (e) and (f) showing screening of inoculum-1, 2 and 3 dipped transformants respectively, on antibiotic irrigated soil filled pots.

Out of four types of inoculums, inoculum- 3 gave best result to produce increased number of transformants. $12 \%-14 \%(18 \pm 4$ antibiotic resistant plants from 100 seeds) increase in the number of transformants on kanamycin plate was observed than the standard inoculums used (Figure 2(c)). 0.075\% silwet L-77 was considered best to get increased number of tranformants. $\sim 150$ seeds were taken from each type for screening on kanamycin plates. Only $2 \%-3 \%$ seeds from standard inoculum (inoculum-1) dipped $\mathrm{T}_{0}$ plants were germinated on kanamycin plate. Inoculum-2 Dipped plants resulted $10 \%-12 \%(14 \pm 4$ antibiotic resistant plants from 100 seeds) increase in transformants (Figure 2(b)). Inoculum-4 dipped $\mathrm{T}_{0}$ plants were wilted after $7-8 \mathrm{hrs}$ of incubation in $28^{0} \mathrm{C}$. Screening of $\mathrm{T}_{0}$ seeds on containing kanamycin agropeat filled pot gave similar result as on kanamycin plates. Increase in transformation efficiency was observed by screening on kanamycin containing agro-peat filled pots (Figures 2(d), (e) and (f)).

Segregation and copy number analysis of transgene in inoculum-3 dipped $T_{2}$ plants compared to inoculum-1 dipped $\mathrm{T}_{2}$ transenics:

PCR analysis of genomic DNA from $T_{1}$ and $T_{2}$ generation plants revealed a single gene segregation pattern of kanamycin registant gene in the Arabidopsis genome. Kanamycin registant gene was not detected in $3(50 \%)$ out of 6 from inoclum 1 dipped $\mathrm{T}_{1}$ progeny where as only $3(20 \%)$ out of 17 , showed kanamycin negative from inoculum- 3 dipped $T_{1}$ progeny. Only one line out of 3 of $\mathrm{T}_{2}$ generation from inoculum-1 dipped transgenic progeny gave homozygous condition (Figure 3(a)). 9 plant out of 17 from $T_{1}$ generation of inoculum- 2 dipped $T_{0}$ plants considered kanamycin positive and 4 plants were detected homozygous in $\mathrm{T}_{2}$ generation (Figure 3(b)). $\mathrm{T}_{1}$ generation of inoculum-3 dipped plants gave 14 transgenic lines detected kanamycin positive and 9 out of them were detected homozygous (Figure 3(c)).

The only transgenic \#2 of $T_{1}$ generation, resulted by inoculum-1 dipping was considered, having 4 copies of transgene and the ratio of the PCR band intensity of the transgene and control gene was found to be 4.2 (Figure 4(a)). The other two (\#1 and\#3) were considered heterozygous as the PCR band intensity varies between target and control gene. From nine transgenic $\left(\mathrm{T}_{1}\right)(\# 1$, $\# 2$, \#3, \#5, \#8, \#9, \#10, \#14 and \#15) lines of inoculum-2 dipped plants, 4 lines (\#1, \#2 and \#10) considered, having single copy of transgene (Figure 4(b)). The ratio between transgene and control gene were found to be 1.2, 0.9 , and1.1 respectively. From forteen transgenic $\left(\mathrm{T}_{1}\right)$ $(\# 2, \# 3, \# 4, \# 5, \# 7, \# 8, \# 9, \# 10, \# 11, \# 12, \# 14, \# 15, \# 16$ and \#17) lines of inoculum-3 dipped plants, 4 lines (\#3, 
Totransgenic Transformants produecd by dipping in inoculum1

line

$\mathrm{T}_{1}$ transgenic

line

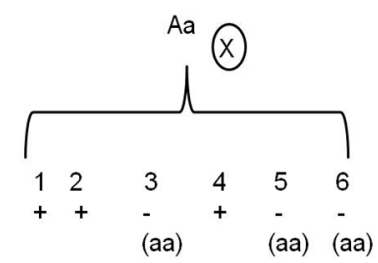

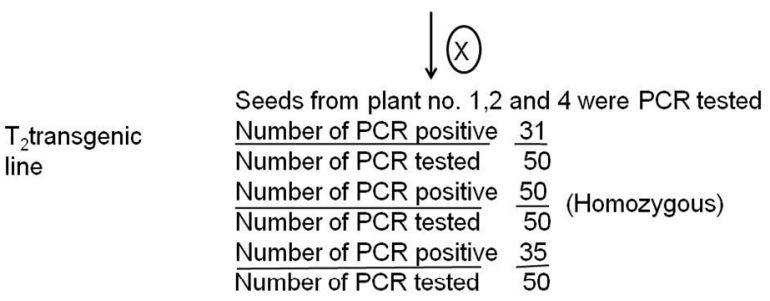

(a)

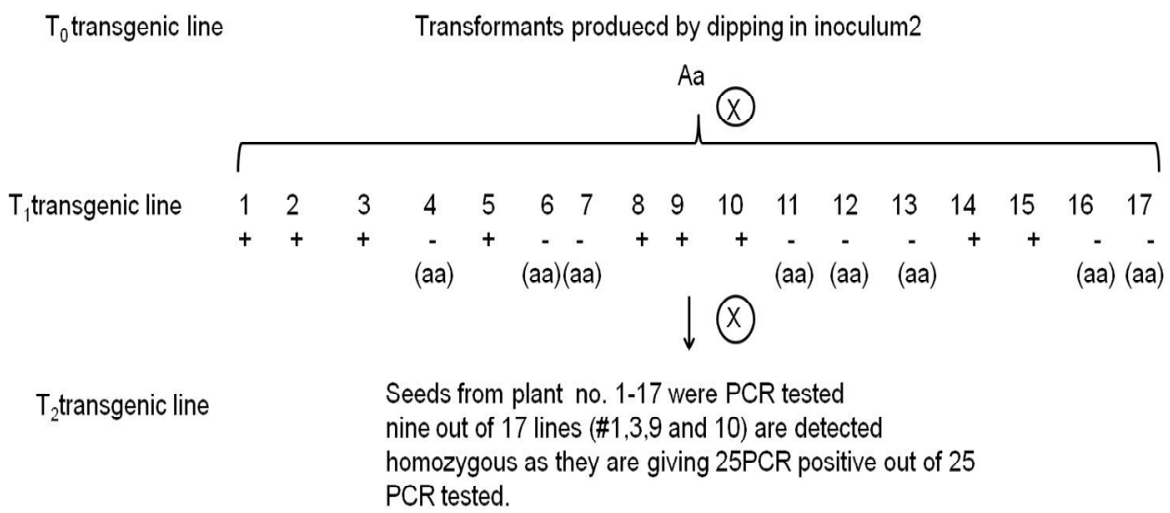

(b)

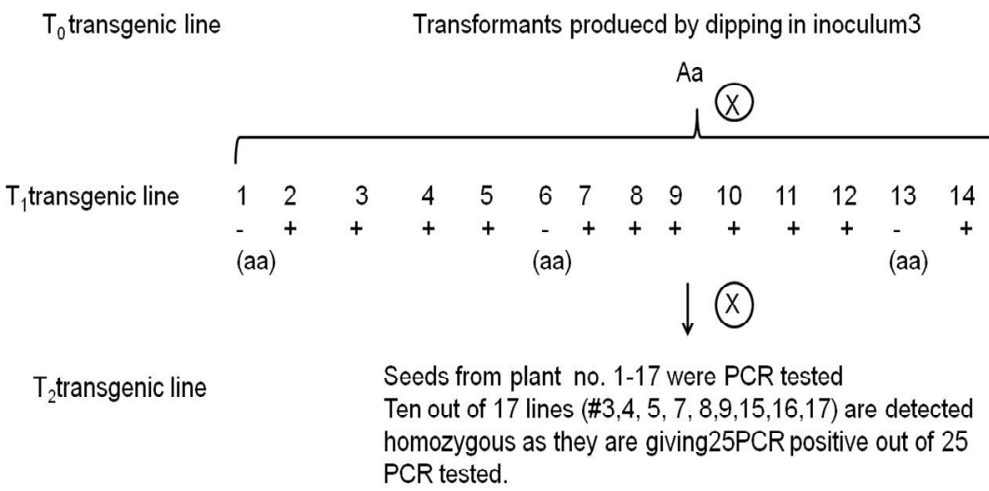

(c)

Figure 3. Segregation (survival) analysis of kanamycin resistant gene in transgenic plants developed using Inoculum-1 (a), inoculum-2 (b) and inoculum-3 (c). Zygosity of transgenic plants, were identified in $\mathrm{T}_{2}$ generation by PCR amplification of integrated gene. $\mathrm{X}$ indicated self pollination. '+' indicated PCR positive and '-' indicated PCR negative transgenic plants.

$\# 4$, \#5, \#7, \#8 and \#9) considered, having single copy of transgene (Figure $\mathbf{4 ( b ) )}$. The ratio between transgene and control gene were found to be $0.9,1.3,1.1,0.9,1.0$ and 1.1 respectively.

\section{DISCUSSION}

Here we confirmed the crucial role of silwet L-77 in the inoculum in enhancement of rate of transgenic produc- 
(a)

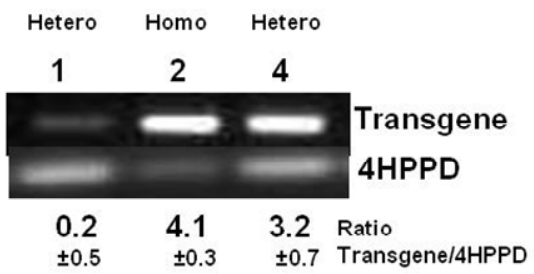

(b)

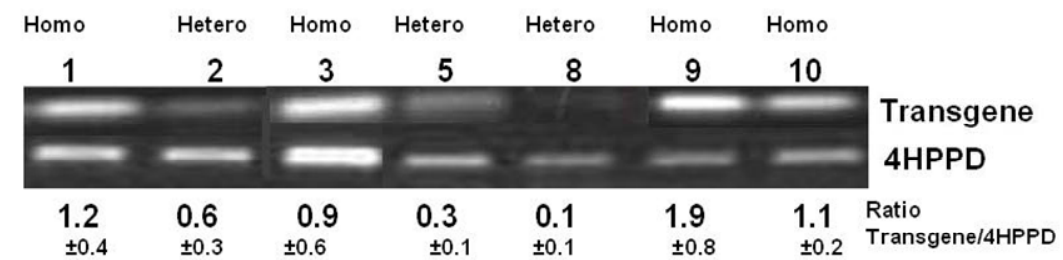

(c)

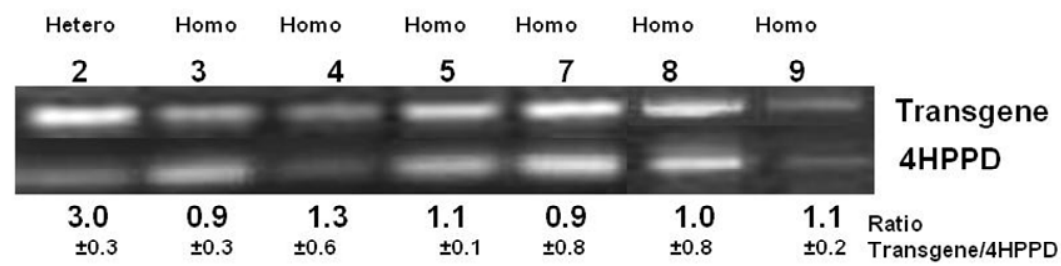

Figure 4. Identification of transgenic Arabidopsis plants carrying a single copy of integrated T-DNA resulted by dipping in inoculum-1 (a), inoculum-2 (b) and inoculum-3 (c) by single step PCR. The ratios of transgene to control (4HPPD) genes were obtained using gel images ( \pm SD indicated). The upper bands represent the transgene amplicons. From inoculum- 1 dipped $\mathrm{T}_{1}$ lines, no single copy was detected. From inoculum- 2 dipped $\mathrm{T}_{1}$ lines $\# 1,2$ and 10 detected as single copy transgenic lines. From inoculum-3 dipped $\mathrm{T}_{1}$ lines \#3, 4, 5, 7, 8 and 9 detected as single copy transgenic lines.

tion. We also found the replacement of sucrose by using glucose in the inoculum. We compared the traditional inoculation method of submerging inflorescences in the bacteria suspension [4] with the modified protocol (Figure 1(a)). Cough and Bent [4] described that the flowering stage is suitable for transformation. Desfeux et al [5] demonstrated that ovules are the target for transformation with Agrobacterium. During ovary development, an open structure exists until 3 days before anthesis, when locules are sealed by the stigma. Thus, applying Agrobacterium inoculum to closed floral buds and not opening flowers is important. Martinez-Trujillo et al. [18] developed a modified method i.e. drop by drop application of Agrobacterium suspension to the closed floral buds. This is quite labour consuming. Here, we would like to say that the budding stage can result more transformants by modifying a little in the inoculums and only single plant (Figure 1(a)) or multiple plants per pot can be used for infiltration for efficient transformant production. Dipping of Primary floral buds can be done to get transformants earlier as it avoids clipping of primary inflorescence and lessen the days to get transformed seeds. 500nm OD value of the agrobacteria culture can be increased upto $\sim 2.5$ to get better result of infection. Curtis [19] explained that the surfactant plays an important role in optimizing transformation efficiency of radish. We optimized the transformation efficiency of Arabidopsis by altering the concentration of silwet L-77. Rinsing of $\mathrm{T}_{0}$ plants after $7-8 \mathrm{hrs}$ of infiltration avoids any toxic effect of excess surfactant. This modification produced $8 \%-10 \%$ enhanced number of $\mathrm{T}_{0}$ transformants than the conventional method. Cough [20] said about the use of other surfactants rather than silwet L-77.We also tested another surfactant i. e. tween-20 instead of silwet L-77 which could also able to produce efficient number of transformants (Figure 3(b)). This can help us from economic view point and in case of unavailability of silwet L-77. Use of Glucose in place of sucrose in the inoculum proved better to give efficient transformant. 
One limitation in the identification of transgenic $A$. thaliana lines after floral dip is that the seeds are often internally contaminated with the A. tumefaciens line used. Furthermore, there is often fungal contamination within the seed. Davis et al [21] developed a non-asceptic condition to avoid fungal contamination at the time of screening of $\mathrm{T}_{0}$ transformants by using chromatography papers treated with antibiotics. We desired to establish a protocol for antibiotic selection that could be used under non-aseptic and in soil conditions. As an added return, this would eliminate the time needed to surface-sterilize seed prior to agar culture and also there would no need of chromatography papers. We were successful in identifying transformants on soil by treating the agro-peat with kanamycin in a concentration of $50 \mu \mathrm{g} / \mu \mathrm{l}$ every day up to rosette leaves come up (Figure 2). However, we were convinced that it was possible to screen antibiotic resistance plants under non-sterile soil conditions.

Transgenic plants derived from Agrobacterium mediated transformation often carry multiple copies of integrated T-DNA [22] consequently, integrated gene can become unstable due to enhanced gene silencing as result of multiple copies of the ectopic gene [23] and zygosity is also a factor for stable transgenic line [6].We compared the zygosity and copy number of integrated transgene following $\mathrm{Fu}$ and Ristic [24], taking conventional (inoculum-1 dipped) and modified (inoculum- 3 dipped) $T_{1}$ and $T_{2}$ transformants. Inoculum-3 dipped $T_{0}$ plants produced enhances number of transformants consequently produced more number of homozygous and single copied transgenic lines (Figure 4) than the inoculum-1 dipped $\mathrm{T}_{0}$ plants. Only one PCR positive $\left(\mathrm{T}_{1}\right)$ plant produced by conventional method proved homozygous in $\mathrm{T}_{2}$ generation and it was harboring 4 copies of transgene. From 9 PCR positive $\mathrm{T}_{1}$ lines of inoculun2 dipped plants 4 were considered homozygous $\mathrm{T}_{2}$ generation and from 9 plants 7 were tested for copy number analysis and 3 were proved harboring single copy of transgene (Figure $4(\mathbf{b})$ ). Similarly $14 \mathrm{PCR}$ positive $\mathrm{T}_{1}$ lines of inoculum-3 dipped plants 9 were considered homozygous $\mathrm{T}_{2}$ generation and from 14 plants 7 were tested for copy number analysis and 6 were proved harboring single copy of transgene (Figure 4(c)).

Here we have presented a very simplified method that is strongly efficient in the generation and selection of homozygous, single copied and stable transgenic Arabidopsis plants, which is less time consuming, economically efficient and avoids aseptic condition.

\section{ACKNOWLEDGEMENTS}

Authors are thankful due to the laboratory facility provided by Prof. B. C. Tripathy, JNU, New Delhi. This work was also supported by Prof. S.C. Sabat, Institute of Life Sciences, Bhubaneswar. Dr. B. B. Sahu is acknowledged due to his valuable suggestion.

\section{REFERENCES}

[1] Feldmann, K.A. and Marks, M.D. (1987) Agrobacterium mediated transformation of germinating seeds of Arabidopsis thaliana: a non-tissue culture approach. Molecular Genomics and Geneicst, 208, 1-9. doi:10.1007/BF00330414

[2] Bechtold, N., Ellis, J. and Pelletier, G. (1993) In planta Agrobacterium-mediated gene transfer by infiltration of adult Arabidopsis thaliana plants. C R Acad Sci Paris, Sciences de la vie/Life sciences, 316, 1194-1199.

Bechtold, N., Jolivet. S., Voisin, R. and Pelletier, G. (2003) The endosperm and the embryo of Arabidopsis thaliana are independently transformed through infiltration by Agrobacterium tumefaciens. Transgenic Research, 12, 509-517. doi:10.1023/A:1024272023966

[3] Clough, S.J. and Bent, A.F. (1998) Floral dip: a simplified method for Agrobacterium mediated transformation of Arabidopsis thaliana. Plant Journal, 16, 735-743. doi:10.1046/j.1365-313x.1998.00343.x

[4] Desfeux, C., Clough, S. J. and Bent, A. F. (2000) Female reproductive tissues are the primary target of Agrobacterium-mediated transformation by the Arabidopsis floral-dip method. Plant Physiology, 123, 895-904. doi:10.1104/pp.123.3.895

[5] Bechtold, N., Jolivet, S., Voisin, R. and Pelletier, G. (2003) The endosperm and the embryo of Arabidopsis thaliana are independently transformed through infiltration by Agrobacterium tumefaciens. Transgenic Research, 12, 509-517. doi:10.1023/A:1024272023966

[6] Labra, M. et al. (2004) Genomic stability in Arabidopsis thaliana transgenic plants obtained by floral dip. Theoretical and Applied Genetics, 109, 1512-1518. doi:10.1007/s00122-004-1773-y

[7] Chung, M.H., Chen, M.K. and Pan, S.M. (2000) Floral spray transformation can efficiently generate Arabidopsis transgenic plants. Transgenic Reserch, 9, 471-476. doi:10.1023/A:1026522104478

[8] Cao M.Q. et al. (2000) Transformation of Pakchoi (Brassica rapa L. ssp. chinensis) by Agrobacterium infiltration. Molecular Breeding, 6, 67-72.

doi:10.1023/A:1009658128964

[9] Tague, B.W. (2001) Germ-line transformation of Arabidopsis lasiocarpa. Transgenic Research, 10, 259-267. doi:10.1023/A:1016633617908

[10] Wang, W.C., Menon, G. and Hansen, G. (2003) Development of a novel Agrobacterium mediated transformation method to recover transgenic Brassica napus plants. Plant Cell Report, 22, 274-281. doi:10.1007/s00299-003-0691-9

[11] Harrison, S.J., Mott, E.K., Parsley, K., Aspinall, S., Gray, J.C. and Cottage, A. (2006) A rapid and robust method of identifying transformed Arabidopsis thaliana seedlings following floral dip transformation. Plant Methods, 2, 19. doi:10.1186/1746-4811-2-19

[12] Liu, N.Y., Zhang, Z.F. and Yang, W.C. (2008) Isolation of embryo-specific mutants in Arabidopsis: Plant transfor- 
mation. Methods in Molecular Biology, 427, 91-100. doi:10.1007/978-1-59745-273-1 7

[13] Logemann, E., Birkenbihl, R.P., Ulker, B. amd Somssich, I.E. (2006) An improved method for preparing Agrobacterium cells that simplifies the Arabidopsis transformation protocol. Plant Methods, 2, 16.

doi:10.1186/1746-4811-2-16

[14] Zhang, X., Henriques, R., Lin, S.S., Niu, Q.W. and Chua, N.H. (2006) Agrobacterium mediated transformation of Arabidopsis thaliana using the floral dip method. Nature Protocols, 1, 641-646. doi:10.1038/nprot.2006.97

[15] Dellaporta, S.I., Wood, J. and Hicks, J.B. (1983) A plant DNA mini preparation: Version II. Plant molecular Biology Reporter, 1, 19-21. doi:10.1007/BF02712670

[16] Fu, J., Momcilovic, I., Clemente, T.E., Nersesian, N., Trick, H.N. and Ristic, Z. (2008) Heterologous expression of a plastid EF-Tu reduces protein thermal eggregation and enhances $\mathrm{CO} 2$ fixation in wheat (Triticum aestivum) following exposure to heat stress. Plant molecular biology, 68, 277-288. doi:10.1007/s11103-008-9369-6

[17] Miguel, M.T., Veronica, L.B., Joseluis, C.P. and Luis, H.E. (2004) Improving transformation efficiency of Arabidopsis thaliana by modifying the floral dip method. Plant Molecular Biology Repoter, 22, 63-70. doi:10.1007/BF02773350

[18] Curtis, S.I. and Nam, G.H (2001) Transgenic radish (Raphanus sativus L. longipinnatus Bailey) by floral-dip method-plant development and surfactant are important in optimizing transformation efficiency. Transgenic Research, 10, 363-371. doi:10.1023/A:1016600517293

[19] Cough, J.S. (2005) Floral dip: Agobacterium-mediated germ line transformstion. Methods in Molecular Biology, 286.

[20] Davis, M.A., Hall, A., Millar, J.A., Darrah, C. and Davis, J.S. (2009) Protocol: Streamlined sub-protocols for floral-dip transformation and selection of transformants in Arabidopsis thaliana. Plant Methods, 5, 3. doi:10.1186/1746-4811-5-3

[21] Grevelding, C., Fantes, V., Kemper, E., Schell, J. and Masterson, R. (1993) Single copy T-DNA insertions in Arabidopsis are the predominant form of integration in root derived trnsgenics, where as multiple insertions are found in leaf discs. Plant Moleclar Biology, 23, 847-860. doi:10.1007/BF00021539

[22] Lechtenberg, B., Schubert, D., Forsbach, A., Gils, M. and Schmidt, R. (2003) Inverted repeat T-DNA configuratios nor arrengements of trigger transgene scilencing. Plant Journal, 34, 507-517. doi:10.1046/j.1365-313X.2003.01746.x

[23] Fu, J. and Z, Ristic. (2010) Analysis of transgenic wheat (Triticum aestivum L.) harboring a maize (Zea mays L.) gene for plastid EF-Tu: Segregation pattern, expression and effects of the transgene. Plant Molecular Biology, 73, 339-347. doi:10.1007/s11103-010-9622-7 